\title{
An FPGA-Based Change-Point Detection for 10Gbps Packet Stream
}

\author{
Takuma IWATA $^{\dagger \text { a) }}$, Kohei NAKAMURA ${ }^{\dagger}$, Yuta TOKUSASHI ${ }^{\dagger}$, Nonmembers, \\ and Hiroki MATSUTANI ${ }^{\dagger}$, Member
}

\begin{abstract}
SUMMARY In statistical analysis and data mining, change-point detection that identifies the change-points which are times when the probability distribution of time series changes has been used for various purposes, such as anomaly detections on network traffic and transaction data. However, computation cost of a conventional AR (Auto-Regression) model based approach is too high and infeasible for online. In this paper, an AR model based online change-point detection algorithm, called ChangeFinder, is implemented on an FPGA (Field Programmable Gate Array) based NIC (Network Interface Card). The proposed system computes the change-point score from time series data received from $10 \mathrm{GbE}$ (10Gbit Ethernet). More specifically, it computes the change-point score at the $10 \mathrm{GbE}$ NIC in advance of host applications. It can find change-points on single or multiple streams using a context memory. This paper aims to reduce the host workload and improve change-point detection performance by offloading ChangeFinder algorithm from host to the NIC. As evaluations, change-point detection in the FPGA NIC is compared with a baseline software implementation and those enhanced by two network optimization techniques using DPDK and Netfilter in terms of throughput. The result demonstrates $16.8 \mathrm{x}$ improvement in change-point detection throughput compared to the baseline software implementation. It is corresponding to the $10 \mathrm{GbE}$ line rate. Performance and area overheads when supporting multiple streams are also evaluated.
\end{abstract}

key words: Change-point detection, FPGA NIC, 10GbE

\section{Introduction}

Due to advances in information and communication technology, datasets exchanged over networks are growing rapidly in the size and the number. As the data sets grow, highbandwidth becomes more important for data analysis and pattern recognition. Change-point detection is a method to identify the change-points which are times when the probability distribution of time series changes. Popular applications of the change-point detection are related to a security field [1], such as detecting a sudden increase in traffic volume by computer virus and worm. It is also used in other application fields, such as transaction data, resource management, and trend analysis [2].

In a conventional change-point detection algorithm [3], time series data are divided into two sets at time $t$. Then AR (Auto-Regression) model is built for each set in addition to whole data. Time $t$ is detected as a change-point if a measured error in the whole model is larger than the sum

Manuscript received January 7, 2019.

Manuscript revised May 30, 2019.

Manuscript publicized July 23, 2019.

${ }^{\dagger}$ The authors are with Graduate School of Science and Technology, Keio University, Yokohama-shi, 223-8522 Japan.

a)E-mail: iwata@arc.ics.keio.ac.jp

DOI: 10.1587/transinf.2019PAP0015 of those in the two divided models by a certain level. The computational cost is too high to use it as an online algorithm since this operation is performed for each $t$. ChangeFinder algorithm [4] solves this issue and can be used as an online change-point detection. However, its computational cost is still high to detect change-points from data received via high bandwidth networks, such as $1 \mathrm{Gbps}$ and 10Gbps, due to heavy workload imposed to the host.

In this paper, change-point detection using ChangeFinder algorithm is implemented on an FPGA (Field Programmable Gate Array) based NIC (Network Interface Card)* The proposed system computes the change-point score from time series data received from 10GbE (10Gbit Ethernet). More specifically, ChangeFinder algorithm implemented in the FPGA NIC computes the score in advance of host applications. It can find change-points on single or multiple streams coming from a $10 \mathrm{GbE}$ interface using a context memory. This paper aims to reduce the host workload and improve change-point detection performance by offloading ChangeFinder algorithm from host to the NIC. Xilinx Vivado HLS, a high-level synthesis tool, is used to implement ChangeFinder on the FPGA. As evaluations, change-point detection in the FPGA NIC is compared with a baseline software implementation and those enhanced by two network optimization techniques using DPDK and Netfilter in terms of throughput. The result demonstrates $16.8 \mathrm{x}$ improvement in change-point detection throughput compared to the baseline software implementation, while keeping the same change-point detection accuracy. Performance and area overheads when supporting up to 32,768 streams are also evaluated.

The rest of this paper is organized as follows. Section 2 introduces ChangeFinder algorithm and related FPGAbased accelerators. Section 3 designs the ChangeFinder module and Sect. 4 integrates it in the FPGA NIC. Section 5 extends the design to support multiple streams. Section 6 evaluates the design in terms of area and throughput. Section 7 concludes this paper.

\section{Background}

In statistical analysis and data mining, change-point detection has been used for various purposes, such as step detec-

${ }^{*}$ This paper is an extended version of our workshop paper [5], by supporting change-point detections on multiple streams. 
tion, edge detection, and anomaly detection. Various algorithms have been introduced in the past years. For example, a paper [6] presents an algorithm for online change-point detection based on the normalized maximum likelihood. a paper [7] presents a non-parametric change-point detection algorithm. Among them, since AR model is one of primary approaches to describe time-varying process, in this paper we will focus on those based on AR model for change-point detection on time-series data. In this section, we will start with a conventional change-point detection based on AR model.

\subsection{AR Model: A Conventional Way}

Let $x_{1}^{n}=x_{1}, \ldots, x_{n}$ denote a time-series, and it is divided into $x_{1}^{t}$ and $x_{t+1}^{n}$ by a time point $t$, where $x_{1}^{t}=x_{1}, \ldots, x_{t}$ and $x_{t+1}^{n}=x_{t+1}, \ldots, x_{n}$. Assuming the $k$-th order AR model, the conditional probability density function of $x_{t}$ is given as follows.

$$
p\left(x_{t} \mid x_{t-k}^{t-1}\right)=\frac{1}{(2 \pi)^{d / 2}|\Sigma|^{1 / 2}} \exp \left[-\frac{\left(x_{t}-\omega_{t}\right)^{T} \Sigma^{-1}\left(x_{t}-\omega_{t}\right)}{2}\right],
$$

where $d$ and $\Sigma$ denote the number of data dimensions and a covariance matrix, respectively.

$\omega_{t}$ is given as follows.

$$
\omega_{t}=\sum_{i=1}^{k} \alpha_{i}\left(x_{t-i}-\mu\right)+\mu,
$$

where $\alpha_{1}, \ldots, \alpha_{k}$ and $\mu$ are model parameters.

Let $\hat{\omega}_{t}$ denote an estimated $\omega_{t}$ calculated by Eq. (2) using estimated model parameters. The model fitting error for $x_{1}^{n}$ is thus given as follows.

$$
I\left(x_{1}^{n}\right)=\sum_{t=1}^{n}\left\|x_{t}-\hat{\omega}_{t}\right\|^{2}
$$

Here, time $t$ is detected as a change-point when $I\left(x_{1}^{t}\right)+$ $I\left(x_{t+1}^{n}\right)$ is sufficiently small compared to $I\left(x_{1}^{n}\right)$. Although this method is simple, computation cost is $O\left(n^{2}\right)$ and thus cannot be used for online change-point detection.

\subsection{ChangeFinder Algorithm}

The above mentioned problem is addressed by SDAR (Sequentially Discounting Auto-Regression model learning) algorithm [8]. ChangeFinder algorithm employs SDAR algorithm for the online change-point detection. Its computational cost is much lower than AR model approach. As one of promising applications, for example, a paper [9] utilizes the SDAR-based change-point detection for detecting fraudulent calls. Apache Hivemall [10], which is a machine learning library on Apache Hive, releases a software module of ChangeFinder. Nevertheless its FPGA-based acceleration has not been reported yet.

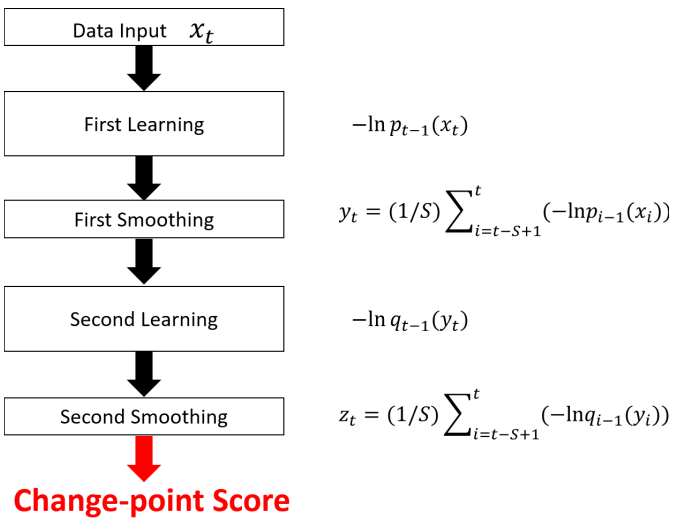

Fig. 1 Flowchart of ChangeFinder

\subsubsection{Overview}

Figure 1 shows the ChangeFinder algorithm that consists of five steps. Each step is described below.

\section{Step 1 (Data Input)}

$x_{t}$ is received at time point $t$.

\section{Step 2 (First Learning)}

For each $t$, an AR model is built. More specifically, a sequence of probability density functions $p_{t}(x): t=1,2, \ldots$ is obtained by the SDAR model, which will be explained later. Please note that $p_{t-1}$ is learned based on $x^{t-1}$. The "outlier" score at $x_{t}$ is calculated as follows.

$$
\operatorname{Score}\left(x_{t}\right)=-\log p_{t-1}\left(x_{t}\right)
$$

\section{Step 3 (First Smoothing)}

For each $t$, a moving average of the outlier scores (obtained in Step 2) in a time window is calculated. More specifically, a sequence of moving averages of the outlier scores $y_{t}: t=0,1,2 \ldots$ is obtained as follows.

$$
y_{t}=\frac{1}{T} \sum_{i=t-T+1}^{t} \operatorname{Score}\left(x_{i}\right),
$$

where $T$ is the length of a time window.

\section{Steps 4 \& 5 (Second Learning \& Smoothing)}

For each $t$, an AR model is built for the new time-series data $y_{t}: t=0,1,2, \ldots$ (obtained in Step 3), and a sequence of new probability density functions $q_{t}(x): t=1,2, \ldots$ is obtained by the SDAR model as well as Step 2. A smoothing step is also applied as well as Step 3. Thus, a sequence of the moving averages $z_{t}: t=0,1,2, \ldots$ is obtained as follows.

$$
z_{t}=\frac{1}{T} \sum_{i=t-T+1}^{t}\left(-\ln q_{t-1}\left(y_{t}\right)\right)
$$

Here, $z_{t}$ is denoted as the "change-point" score at time 

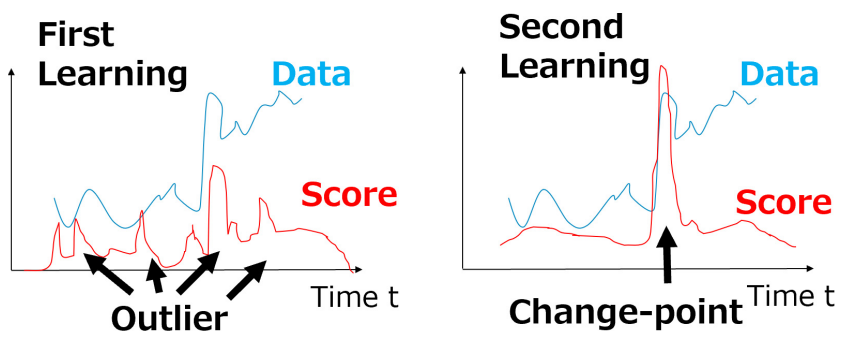

Fig. 2 Two-phase learning of ChangeFinder

$t$. A higher change-point score $z_{t}$ indicates a higher possibility of change-point at time $t$. As shown in Fig. 2, by using the two-phase learning, outliers are eliminated by the first smoothing step and thus only the change-points where the probability distribution of time series changes are extracted.

\subsubsection{SDAR Model}

SDAR model is used for online discounting learning that relies on AR model. ChangeFinder algorithm uses SDAR model to obtain the sequences of probability density functions $p_{t}(x)$ and $q_{t}(x)$. These probability density functions are derived from $\omega_{t}$ and $\Sigma$ in Eq. (1). These parameters are updated by following expressions each time.

$$
\begin{aligned}
\hat{\mu} & :=(1-r) \hat{\mu}+r x_{t} \\
C_{j} & :=(1-r) C_{j}+r\left(x_{t}-\hat{\mu}\right)\left(x_{t-j}-\hat{\mu}\right)^{T} \\
\hat{x}_{t} & :=\sum_{i=1}^{k} \hat{\omega}_{i}\left(x_{t-i}-\hat{\mu}\right)+\hat{\mu} \\
\hat{\Sigma} & :=(1-r) \hat{\Sigma}+r\left(x_{t}-\hat{x}_{t}\right)\left(x_{t}-\hat{x}_{t}\right)^{T}
\end{aligned}
$$

Here, $r$ is a discounting rate. A smaller $r$ indicates a greater influence on past data. For each $t$, an weighted average $\hat{\mu}$ is updated using $r$ and $x_{t}$ in Eq. (7). Based on $C_{j}: j=1, \ldots, k$ obtained in Eq. (8), estimated $\omega_{1}, \ldots, \omega_{k}$ (denoted as $\hat{\omega}_{1}, \ldots$, $\hat{\omega}_{k}$ ) are derived so that the following equation is satisfied.

$$
\sum_{i=1}^{k} \omega_{i} C_{j-i}=C_{j}
$$

Then $\hat{\omega}_{1}, \ldots, \hat{\omega}_{k}$ are used for Eq. (9).

By introducing the discounting effect, SDAR model can be used for online learning on non-stationary time-series data. In addition, the computation cost is reduced down to $O(n)$ and thus it is preferred for online change-point detection.

\subsection{Related Work}

In this paper, change-point detection using ChangeFinder algorithm is implemented on an FPGA NIC that has four $10 \mathrm{GbE}$ interfaces. NPCUSUM (Non-Parametric Cumulative SUM) is a classic and simple change-point detection algorithm. In a paper [11], it is implemented on a high-speed FPGA NIC in order to detect attacks from network. The network attack detection using NPCUSUM is illustrated below.

$$
\begin{aligned}
& S_{0}=0 \\
& S_{n}=\max \left\{0, S_{n-1}+X_{n}-\hat{\mu}-\epsilon \hat{\theta}\right\},
\end{aligned}
$$

where $X_{n}$ denotes input data. $\hat{\mu}$ is an estimated value of $X_{n}$ before an attack, $\hat{\theta}$ is that after the attack, and $\epsilon$ is a tuning parameter. An attack from the network is detected when $S_{n}$ becomes unstable and changes drastically. Although this approach is quite simple to implement, some parameters must be known in advance depending on given applications. In addition the design presented in the paper [11] achieves $100 \mathrm{Gbps}$ when fixed-point values are fed as inputs, while it is $2.5 \mathrm{Gbps}$ when floating-point values are fed. Please note that the proposed ChangeFinder NIC can process floatingpoint inputs at $10 \mathrm{Gbps}$, so it is superior than the paper [11] in terms of throughput when floating-point values are used in the application.

There are some prior works that present FPGA-based outlier detection that detects anomaly values (not changepoints). In a paper [12], for example, an outlier detection based on Mahalanobis distance is implemented in an FPGA NIC. As a more practical outlier detection algorithm, in a paper [13], LOF (Local Outlier Factor) algorithm is accelerated by using an FPGA. Normal data are filtered at the NIC and only anomaly data are transferred to the host machine to reduce data size. In addition, KNN (K-Nearest Neighbor) algorithm is accelerated by using FPGAs in papers [13], [14]. It can be used for outlier detection on timeseries data. Let $X_{t}$ denote input data at time $t$. Among recent data, $k$ nearest neighbors from $X_{t}$ are extracted by $\mathrm{KNN}$ algorithm. If their average distance from $X_{t}$ exceeds a given threshold, then $X_{t}$ is detected as an outlier.

In this paper, the proposed system can find changepoints on multiple streams coming from a 10GbE interface. In other words, we assume multiple time-series data (or multiple streams) which are independent but coming from the same interface. The design highly depends on whether computation result of a single sample is influenced by the previous samples in the same stream. If it is not influenced by the previous samples in the same stream, an incoming sample is simply processed without considering the previous computation result of the same stream; thus a context for each stream is not considered.

On the other hand, if a computation result is influenced by the previous samples in the same stream, a context must be maintained for each stream. A straightforward approach for such cases is to increase the number of stream processing cores so that each core is in charge of a single stream. In this case, workload can be distributed to multiple cores. In a paper [15], an FPGA-based FFT processing for variable length and multiple streams is proposed by using this method. In a paper [16], a compression mechanism for floating-point numerical data streams is proposed by using FPGA. In these cases, multiple instances are introduced to handle multiple streams. The number of instances should be carefully selected by considering the expected number of streams.

Another approach is to handle multiple streams using a single instance. In this case, a context for each stream 
is maintained, and it is switched depending on the sample data currently being processed. For the context switching, a context of the current stream is stored and then that of the next stream is loaded. In papers [17], [18], a regular expression matching mechanism for virus detection is implemented by using FPGA, and context memories for storing context data to support multiple streams are implemented by using distributed RAMs or external memory. In this paper, we employ this approach to find change-points on multiple streams.

Although our target is change-point detection to detect trend changes, ChangeFinder algorithm can be used for both the change-point detection and outlier detection. Actually, the result of the first learning phase $S \operatorname{core}\left(x_{t}\right)$ is used as outlier score, while the final output $z_{t}$ is used as changepoint score. Please note that this paper is the first work that accelerates ChangeFinder algorithm that supports both the change-point and outlier detections by using FPGA NIC.

\section{ChangeFinder on FPGA}

ChangeFinder module on FPGA is illustrated in this section. It is integrated into an FPGA NIC in Sect. 4. ChangeFinder module is written in C. As a high-level synthesis tool we use Xilinx Vivado HLS for the implementation.

\subsection{Pipeline Structure}

Figure 3 illustrates an overview of ChangeFinder module. It consists of pipelined six stages as mentioned in Sect. 2.2. As input data, a 32-bit float value is fed to the module. It is processed as follows.

- sdar1: A probability density function $p_{t}(x)$ for input data $x_{t}$ in the first learning phase is computed.

- $\log 1$ : A logarithmic loss of the probability density function is computed as an outlier score.

- smooth1: A moving average $y_{t}$ of the outlier scores is computed as a result of the first learning phase.

- sdar2: A probability density function $q_{t}(x)$ for $y_{t}$ in the second learning phase is computed.

- $\log 2$ : A logarithmic loss of the probability density function is computed as a log loss score.

- smooth2: A moving average $z_{t}$ of the log loss scores is computed as a change-point score.

These stages operate at $125 \mathrm{MHz}$. In Fig. 3, the number in each pipeline stage indicates the minimum interval between two input data in the stage. For example, "1clk" indicates that new data can be accepted in every cycle. Thus, $\log 1$, smooth $1, \log 2$, and smooth 2 can accept new data every cycle, while sdarl and sdar2 accept new data in every eight cycles. Please note that sdar 1 and sdar2, $\log 1$ and $\log 2$, and smooth1 and smooth 2 are identical, respectively. Each module is pipelined by the HLS PIPELINE directive. The input interval values in Fig. 3 represent the values obtained by this pipelining. Also, the memory in each module is optimized by the HLS ARRAY_PARTITION directive. The

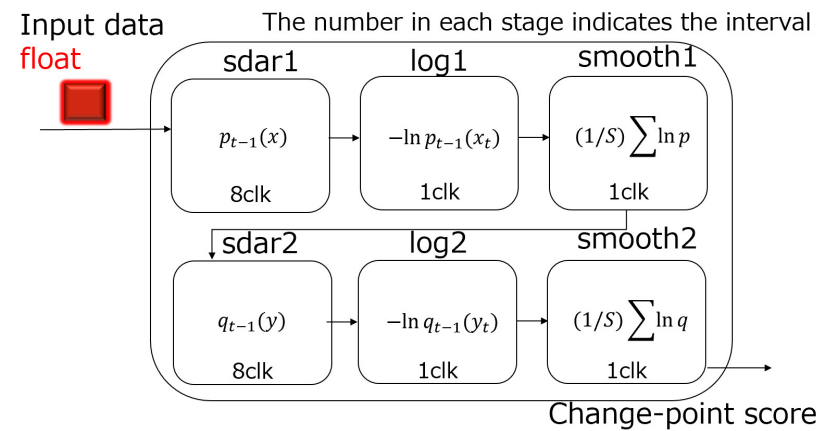

Fig. 3 Pipeline of ChangeFinder module

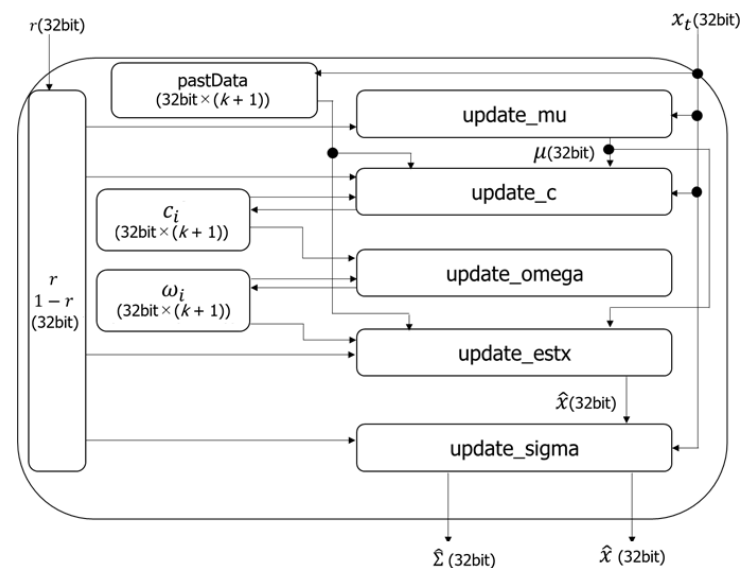

Fig. 4 sdar module

input interval of $s d a r 1$ and $s d a r 2$ modules is currently eight cycles. This is because update_omega submodule of sdarl and sdar 2 modules takes eight cycles to process each input data. Further optimization of the input interval is our future work. In the following, sdarl, $\log 1$, and smooth1 modules are illustrated.

\subsection{SDAR Module}

Figure 4 shows sdar module. Its inputs are $r$ and $x_{t} . r$ is a discounting parameter. Based on it, $(1-r)$ is computed. $x_{t}$ is an input float value. The outputs are $\hat{x}$ and $\hat{\Sigma}$. $\hat{x}$ is an estimated value of $x_{t}$ and $\hat{\Sigma}$ is that of $\Sigma_{t}$.

As shown, sdarl is further divided into five pipelined submodules: update_mu, update_c, update_omega, update_estx, and update_sigma. $x_{t}$ is stored in $(k+1)$ 32-bit registers (pastData in the figure) to refer to past $k$ data, where $k$ is the order of AR model. Similarly, $C_{i}$ and $\omega_{i}$ are accumulated in $(k+1)$ 32-bit registers, respectively.

$x_{t}, r$, and $(1-r)$ are fed to update $-m u$ submodule. It is corresponding to Eq. (7) and computes $\mu$. $\mu$ is then fed to $u p$ date_c and update_est $x$ submodules. update_c submodule is corresponding to Eq. (8) and updates $C_{i}$ registers. Using the updated $C_{i}$ registers, update_omega submodule updates $\omega_{i}$ registers based on Eq. (11). update_estx submodule is corresponding to Eq. (9). Using the updated $\omega_{i}$ registers, pastData registers, and $\mu$, it computes $\hat{x}_{t}$. Finally, update_sigma 
submodule is corresponding to Eq. (10). Using $\hat{x}_{t}$ and $x_{t}$, it computes $\hat{\Sigma}$.

These five submodules work in a pipelined manner. As a result, sdarl module accepts new data $x_{t}$ in every eight cycles.

\subsection{Log and Smooth Modules}

Regarding $\log 1$ module, its inputs are $\hat{x}_{t}, \hat{\Sigma}$, and $x_{t}$ in a $32-$ bit float format. The 32-bit output is used as an outlier score (for $\log 2$, it is used as a $\log \operatorname{loss}$ score). It performs a logarithmic computation as in Eq. (4). It is fully pipelined and can accept new data in every cycle.

Regarding smoothl module, its inputs are a window size $T$ and the 32-bit outlier score from $\log 1$ module (for smooth2, it is the 32-bit log loss score from $\log 2$ module). The 32-bit output is the result of the first learning phase $y_{t}$ (for smooth2, it is the change-point score $z_{t}$ ). The input score is stored in $(T+1)$ 32-bit registers to refer to past $T$ data. Then it computes a moving average of recent $T$ data as in Eq. (5). The maximum $T$ is set to eight in our design. It is also fully pipelined and can accept new data in every cycle.

\section{ChangeFinder on FPGA NIC}

ChangeFinder module is implemented on a $10 \mathrm{GbE}$ FPGA NIC. It is denoted as ChangeFinder NIC in this paper. It performs change-point detection for each numerical value coming from the $10 \mathrm{GbE}$ network. The change-point score computed at the NIC is passed to a host application so that it can identify changes in given time series data. Users can give some parameters (i.e., AR model order $k$, discounting rate $r$, and smoothing window size $T$ ) to the ChangeFinder NIC via ioctl so that the users can tune ChangeFinder module suitable for time series data of the target application.

In this paper, NetFPGA-SUME [19] is adopted as a $10 \mathrm{GbE}$ FPGA NIC. It has four $10 \mathrm{GbE}$ interfaces. Packets received by these interfaces are processed at an on-board FPGA and the results are transferred to a host machine via a PCI-Express Gen 3 x8 interface. We use 10GbE MAC IP core provided by Xilinx. We also use Reference NIC design provided by NetFPGA project [20] as a standard $10 \mathrm{GbE}$ NIC function. ChangeFinder module is inserted along the datapath of Reference NIC design.

We implemented a wrapper module along the datapath of Reference NIC design so that all the received packets go through the wrapper module. Then ChangeFinder module designed with Xilinx Vivado HLS is implemented inside the wrapper module. Figure 5 shows a block diagram of ChangeFinder NIC consisting of ChangeFinder module and Reference NIC. In Reference NIC, packets received by the four $10 \mathrm{GbE}$ interfaces (i.e., RX0 to RX3) and host DMAC are arbitrated at Input Arbiter module. Then, an output port is selected among the four $10 \mathrm{GbE}$ interfaces (i.e., TX0 to TX3) and host DMAC for each packet. Packets are stored and transmitted via BRAM Output Queues corresponding

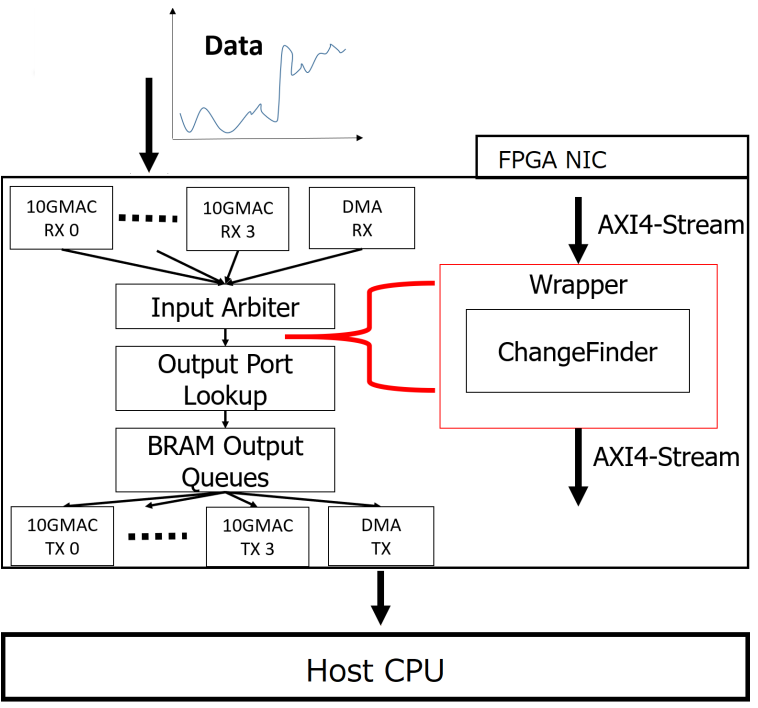

Fig. 5 ChageFinder on FPGA NIC

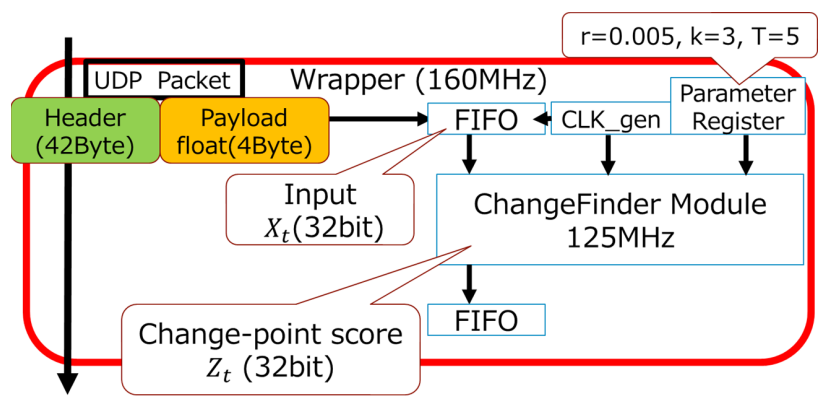

Fig. 6 Connection between wrapper and ChangeFinder modules

to the selected output ports. Packets are transferred between these modules as AXI4 stream [21]. The wrapper module is implemented between Input Arbiter and Outport Lookup modules. We use UDP/IP as transport/network layer protocols. ChangeFinder module computes a change-point score for each incoming packet destined to a specific UDP port. All the other packets including ARP and ICMP just skip the wrapper module without any additional delay.

Figure 6 illustrates the wrapper module and input/output signals of ChangeFinder module. Their connection complies with AXI4 standard. A clock generator of $125 \mathrm{MHz}$ and parameter registers are implemented for ChangeFinder module. In addition, an input asynchronous FIFO buffer is inserted between them. Because ChangeFinder module is operating at $125 \mathrm{MHz}$ and Reference NIC is operating at $160 \mathrm{MHz}$, the input FIFO buffer is used to absorb their different clock frequencies.

The wrapper module identifies packets that contain sample data. Then it extracts the sample data and feeds them to ChangeFinder module. The packet conveys sample data $x_{t}$ in a 32-bit float format in a UDP payload. UDP packets with a specific destination port number are extracted as sample packets and they are fed to the input FIFO buffer. As tuning parameters, AR model order $k$, discounting rate 
$r$, and smoothing window size $T$ are stored in the parameter registers. They are fed to ChangeFinder module in addition to input data $x_{t}$ when ChangeFinder module is ready. Then the change-point score $z_{t}$ is computed and fed to an output asynchronous FIFO buffer. The score $z_{t}$ can be embedded in the original packet and passed to host application. It is also stored in a register inside the wrapper module which can be accessed by the host application via ioctl.

\section{Extension for Multiple Streams}

ChangeFinder module illustrated so far is designed to find change-points on a single stream. In this section, it is extended to support multiple streams coming from a $10 \mathrm{GbE}$ interface. Actually, it is practical to assume that multiple streams from one or more sources are fed to the proposed ChangeFinder NIC. We are assuming some applications for this implementation, such as a server that aggregates sensor values from many sensor nodes. It can be applied to an intrusion detection system that monitors many network flows between host and specific IP addresses. The change-point detection requires a context for each stream, because computation result of a sample is influenced by the previous results in the same stream. As discussed in Sect. 2, a straightforward design is to replicate the ChangeFinder module so that each module is in charge of a single stream. However, as shown in Sect. 6.3, the number of instances which can be implemented on the FPGA NIC is at most eight, while the number of streams can be easily increased depending on the number of stream sources. In this section, we thus extend the ChangeFinder module so that a single instance can support multiple streams by using a context memory to store context of each stream.

In our design, a fine-grained context switching, where a context switching occurs in each pipeline stage separately, is implemented. Such a fine-grained context switching is beneficial compared to the "Run-to-Completion" approach, where context of all the pipeline stages is switched at the same time, in terms of context switching latency. Figure 7 shows the multi-stream version assuming $k=2$ and $T=8$. There are a BRAM-based context memory and six stages: sdar1, $\log 1$, smooth1, sdar2, $\log 2$, and smooth2.

Each stage identifies the stream ID of the sample currently being processed. It loads and saves the necessary context from/to the context memory based on the stream ID currently being processed. That is, the stream ID is used as address of the context memory.

Context data for all the streams must be maintained, while the number of streams can be easily increased depending on the number of stream sources; thus context data size should be minimized. Context data size depends on the order of AR model $k$ and the smoothing window size $T$. Table 1 lists context data fields and their sizes when $k=2$ and $T=8$. In this case, the context size for each stream is 76 bytes. Assuming Xilinx Virtex-7 XC7VX690T FPGA is used for the implementation, because its BRAM capacity is about 52.9 Mbits, up to 87,006 streams can be stored in

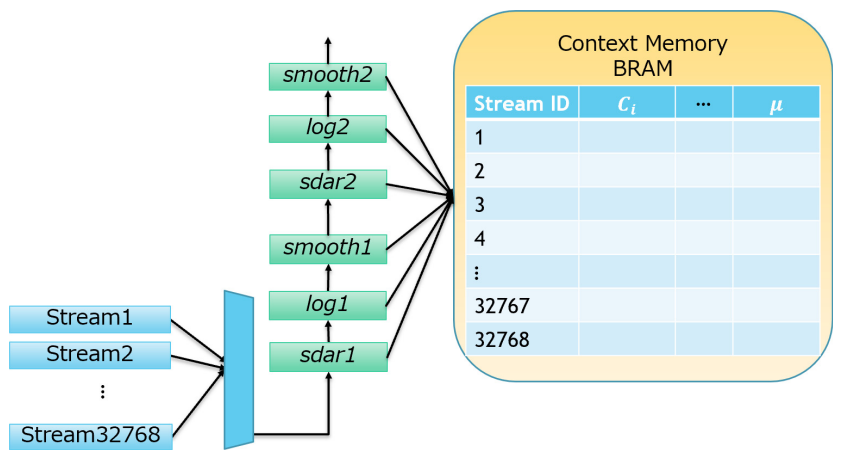

Fig. 7 ChangeFinder module for multiple streams

Table 1 Context data for each stream

\begin{tabular}{c|c|c|c|c|c|c|c|c}
\hline \hline Data & $C_{i}$ & $\Sigma$ & pastData & $\mu$ & $r$ & $\omega$ & index & count \\
\hline Size (byte) & 12 & 4 & 32 & 4 & 4 & 12 & 4 & 4 \\
\hline
\end{tabular}

the BRAM. Please note that only 32,768 streams could be implemented in total after the placement and routing were done. This is because the NIC part also consumes BRAM. If the number of streams is less than the BRAM capacity, a fast context switching can be simply implemented without any external memories.

\section{Evaluations}

\subsection{Preliminary Evaluations of Parameters}

Context data size depends on the order of AR model $k$ and the smoothing window size $T$, so we conduct two preliminary evaluations using real datasets in order to find appropriate parameters of ChangeFinder.

First we apply ChangeFinder to a network intrusion detection. We use CICIDS2017 dataset [22] provided by University of New Brunswick. This dataset consists of labeled network flows during five days. We use Wednesday dataset which includes various DDoS attacks, because papers [8] and [23] show that detection of DDoS attack is one of applications for their change-point detection algorithm. The rate of SYN flag and FIN flag which is updated every second is used as input for ChangeFinder. $r$ is set to 0.001 . The evaluation results while changing $k$ and $T$ are shown in Figs. 9 and 10.

Figure 9 shows the result when $k$ is changed to 2, 3, and 4 while $T$ is fixed to 3 . The blue line represents the input value, and the green line represents the change-point score. $\mathrm{X}$-axis shows the elapsed time and the time unit is one second. The result shows that the point at which the change-point score becomes high is almost the same in these $k$ values, and it is not necessary to set $k$ larger than 2 for this dataset.

Figure 10 shows the result when $T$ is changed to 3,4 , and 8 while $k$ is fixed to 2 . Since $T$ is the smooth window size, the change-point score decreases as $T$ increases.

Secondly we apply ChangeFinder to analyze stock data. We use TOPIX (Tokyo stock price index) data [24] 


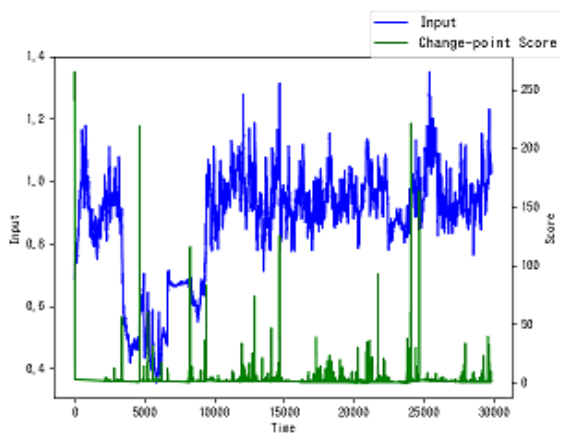

(a) $k=2, T=3$

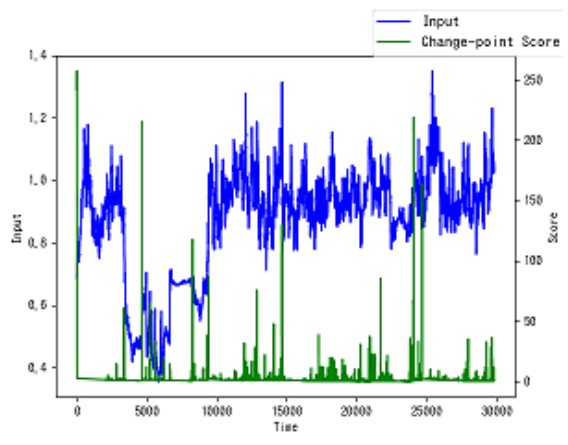

(b) $k=3, T=3$

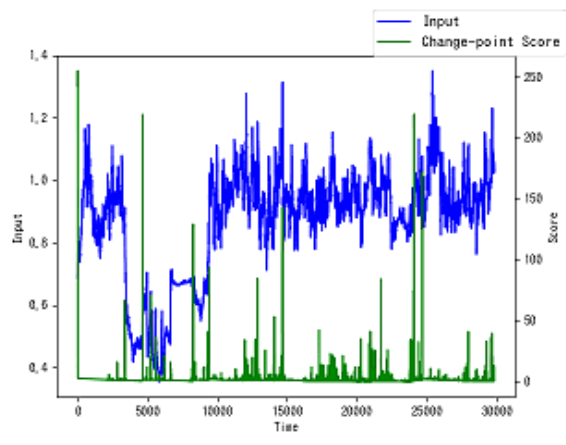

(c) $k=4, T=3$

Fig. 9 Behavior of change-point score when $k$ is changed (IDS dataset)

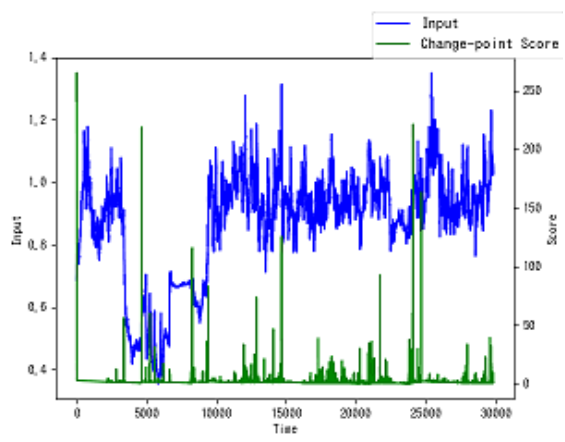

(a) $k=2, T=3$

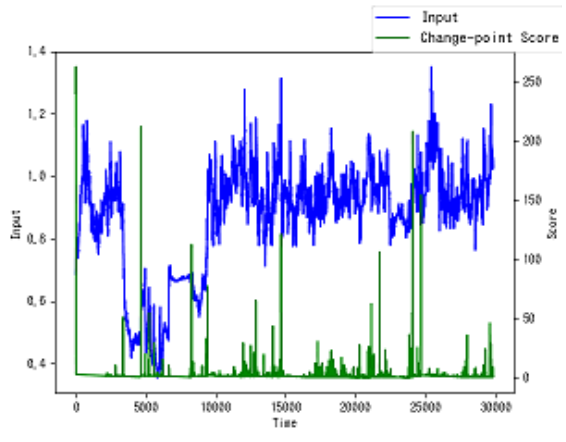

(b) $k=2, T=4$

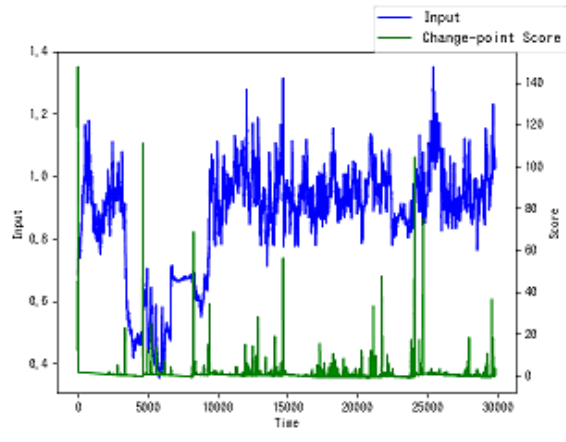

(c) $k=2, T=8$

Fig. 10 Behavior of change-point score when $T$ is changed (IDS dataset)

from 1987 to 2019. $r$ is set to 0.005 . The evaluation results while changing $k$ and $T$ are shown in Figs. 11 and 12. X-axis shows the elapsed time and the time unit is one day.

As far as the two evaluation results are considered, it can be said that $k$ is enough high even at around 2. On the other hand, $T$ depends on the sensibility of the input value and how much the change is ignored as an outlier. There was no significant differences in the number of change points in these evaluations. Please note that the maximum $T$ must be determined when ChangeFinder core is synthesized. In the current implementation, the maximum $T$ is set to 8 which is the largest value confirmed in the preliminary evaluations.

\subsection{Evaluation Environment}

The target 10GbE FPGA NIC is NetFPGA-SUME that has a Xilinx Virtex-7 XC7VX690T FPGA and four SFP+ 10GbE interfaces. It is mounted to a host machine via PCI-Express Gen3 x8 interface. We use Xilinx Vivado HLS version 2016.4 for the implementation. Reference NIC part is operating at $160 \mathrm{MHz}$, while the proposed ChangeFinder module is running at $125 \mathrm{MHz}$ in the case of single-stream design.

Figure 8 shows the evaluation environment using two machines and Table 2 shows their specification. In the throughput measurement, if a software program at the client machine generates time series data and sends them to the server, there is a possibility that the client cannot fully uti-

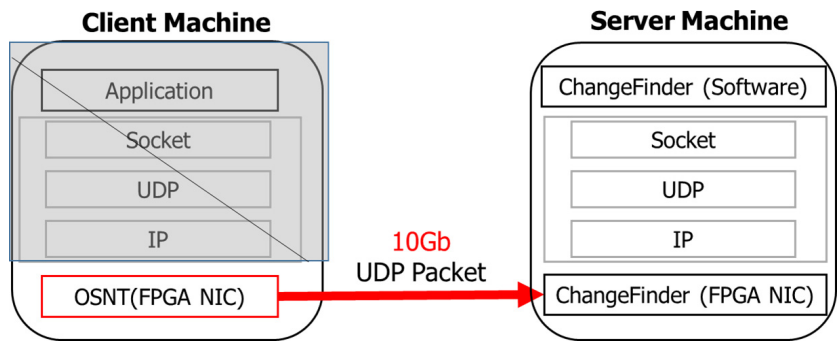

Fig. 8 Evaluation environment for throughput

Table 2 Machines used in the environment

\begin{tabular}{c|c|c}
\hline \hline & Server (host) machine & Client machine \\
\hline CPU & Intel Core i5-4460 & Intel Core i5-4460 \\
OS & Ubuntu 14.04 & CentOS 6.6 \\
NIC & NetFPGA-SUME (Proposal) & NetFPGA-10G for OSNT \\
& Intel X520-DA2 (Software) & \\
\hline
\end{tabular}

lize the $10 \mathrm{GbE}$ bandwidth. Therefore, we used a hardware packet generator, called Open Source Network Tester (OSNT), as the client. OSNT is implemented on the FPGA NIC and can generate packets at the throughput of $10 \mathrm{GbE}$ line rate. The client and server machines are connected by a $\mathrm{SFP}+$ direct attached cable for 10GbE. The client machine has an FPGA NIC with OSNT installed, and sends packets to the server. In the server machine, the proposed ChangeFinder module is implemented on the FPGA NIC and pro- 


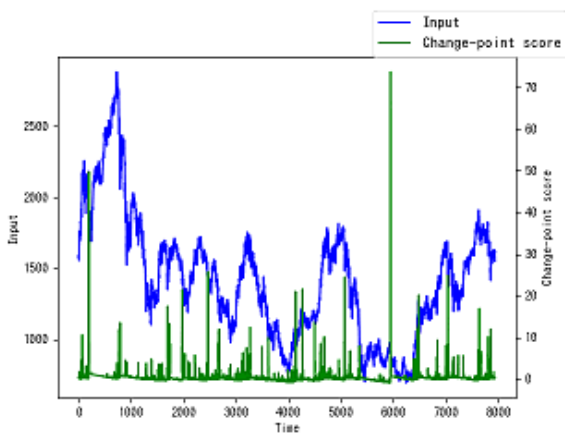

(a) $k=2, T=3$

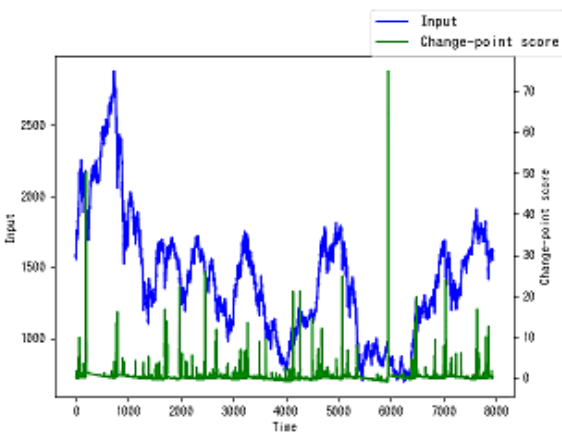

(b) $k=3, T=3$

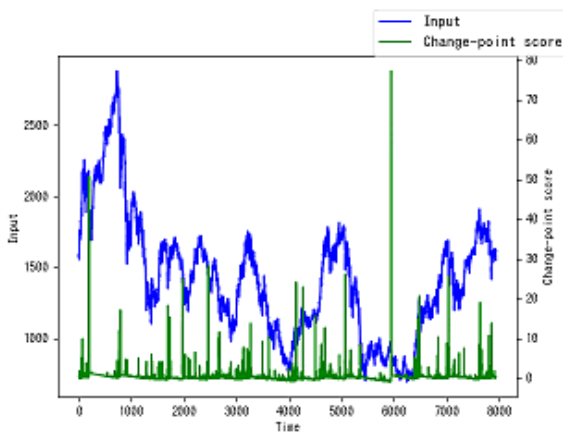

(c) $k=4, T=3$

Fig. 11 Behavior of change-point score when $k$ is changed (TOPIX dataset)

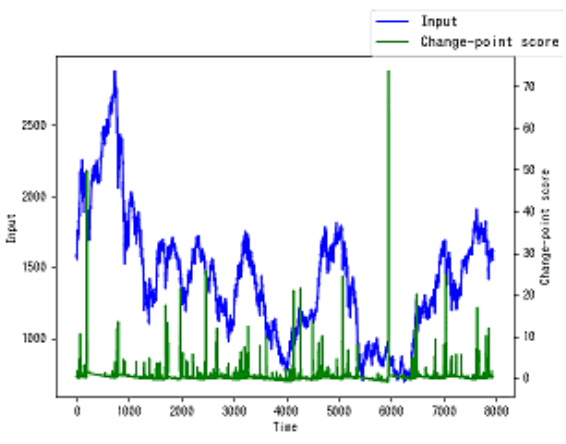

(a) $k=2, T=3$

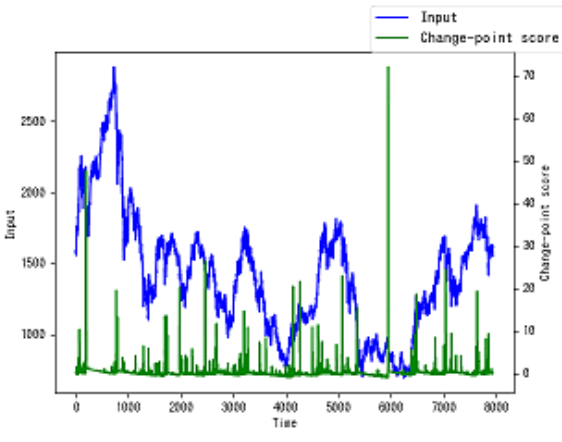

(b) $k=2, T=4$

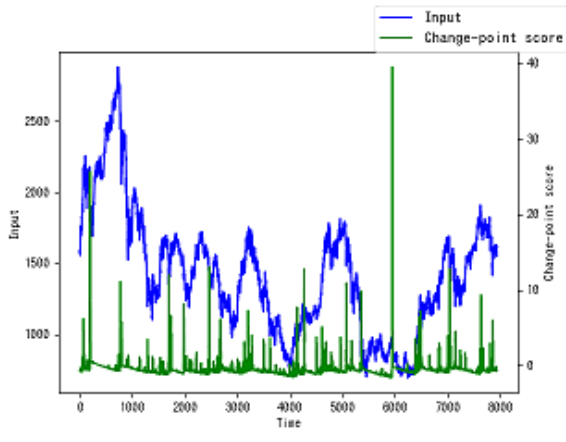

(c) $k=2, T=8$

Fig. 12 Behavior of change-point score when $T$ is changed (TOPIX dataset)

cesses incoming time series data. We measured the number of sample data processed at the ChangeFinder module per a second as throughput.

\subsection{Resource Utilization}

In this section, first, ChangeFinder module for a single stream is evaluated in terms of resource utilization. That for multiple streams is then evaluated.

Table 3 shows DSP, FF, and LUT utilizations of submodules (i.e., sdar1, $\log 1$, smooth1, sdar2, $\log 2$, and smooth2) in the proposed ChangeFinder module for a single stream. Table 4 shows those of whole ChangeFinder NIC including ChangeFinder module and Reference NIC. Although $\log 1$ and $\log 2$ submodules that perform logarithm computations in parallel consume more resources than the others, their resource utilizations are still low. As shown in Table 4, ChangeFinder module consumes up to $12.1 \%$ of the FPGA resources. Even with $10 \mathrm{GbE}$ NIC functionality, the entire resource utilizations are less than or equal to $18.8 \%$. Regarding the processing time, it takes 347 cycles to complete all the processing for a single sample, so the latency of all the processing is $2.78 \mu \mathrm{sec}$.

Although up to eight ChangeFinder modules can be implemented on the FPGA according to Table 4, in this paper, as shown in Sect. 5, we do not increase the number of ChangeFinder modules but increase the capacity of a single
Table 3 Resources used in single-stream ChangeFinder module

\begin{tabular}{c|c|c|c|c|c|c}
\hline \hline & sdar1 & $\log 1$ & smooth1 & sdar2 & log2 & smooth2 \\
\hline DSP & 39 & 122 & 14 & 39 & 122 & 14 \\
FF & 5,426 & 9,070 & 2,777 & 5,426 & 9,070 & 2,777 \\
LUT & 5,202 & 11,491 & 2,864 & 5,202 & 11,491 & 2,864 \\
\hline
\end{tabular}

Table 4 Resources used in single-stream ChangeFinder NIC

\begin{tabular}{c|c|c}
\hline \hline & ChangeFinder & ChangeFinder + Reference NIC \\
\hline DSP & $437(12.1 \%)$ & $437(12.1 \%)$ \\
FF & $35,081(4.6 \%)$ & $100,403(11.6 \%)$ \\
LUT & $37,131(8.5 \%)$ & $81,517(18.8 \%)$ \\
BRAM & $0(0 \%)$ & $198(13.5 \%)$ \\
\hline
\end{tabular}

ChangeFinder module so that it can support many streams. In this case, the size of the BRAM-based context memory increases as the maximum number of streams increases. Figure 13 shows the BRAM utilization when increasing the maximum number of streams. Up to 32,768 streams can be implemented on the BRAM. When increasing the number of streams from one to 32,768 , the operating frequency dropped from $125 \mathrm{MHz}$ to $80 \mathrm{MHz}$. When 32,768 streams are implemented, the operating frequency is $80 \mathrm{MHz}$ and the number of clock cycles to process a single sample is 260 , so the processing latency is $3.25 \mu \mathrm{sec}$, which is $0.47 \mu \mathrm{sec}$ longer than the original design that supports a single stream. Table 5 shows the resource utilizations of ChangeFinder NIC that supports 32,768 streams. 


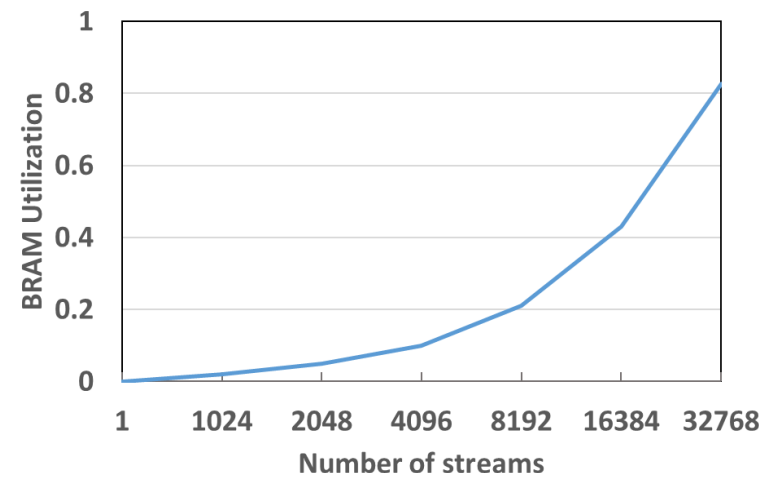

Fig. 13 BRAM utilization for multiple streams

Table 5 Resources used in multi-stream ChangeFinder NIC

\begin{tabular}{c|c|c}
\hline \hline & ChangeFinder & ChangeFinder + Reference NIC \\
\hline DSP & $376(10.4 \%)$ & $376(10.4 \%)$ \\
FF & $25,213(3.3 \%)$ & $89,659(10.4 \%)$ \\
LUT & $36,766(8.4 \%)$ & $80,470(18.6 \%)$ \\
BRAM & $1,216(82.7 \%)$ & $1,414(96.2 \%)$ \\
\hline
\end{tabular}

BRAM utilization is $96.2 \%$, while the other resource utilizations are less than $18.6 \%$. FF utilization is lower than that of the single-stream design since a part of FFs are replaced with BRAMs. The other resource utilizations, such as DSP and LUT, are also reduced because of a relaxed timing constraint targeting $80 \mathrm{MHz}$.

\subsection{Throughput}

As mentioned above, OSNT at the client machine transmits time-series data at $10 \mathrm{GbE}$ line rate to the server machine, and the number of sample data processed within one second at the server machine is measured as throughput.

The proposed ChangeFinder NIC is compared with three software-based counterparts implemented in C: Baseline, DPDK, and Netfilter. In Baseline, a ChangeFinder program is running on the application layer. A standard UDP/IP processing is performed by Linux kernel for each sample data. In DPDK, although the ChangeFinder program is running on the application layer, the program directly accesses the NIC without kernel UDP/IP stack. In Netfilter, the ChangeFinder program is implemented as a kernel module using a Linux Netfilter framework. Please note that a float value is approximated as a fixed-point format in the Netfilter version. In all these cases, a single-stream ChangeFinder is used for the measurement.

Figure 14 shows their throughputs. The throughput of our ChangeFinder module is denoted as FPGA(sim) and the ChangeFinder NIC consisting of ChangeFinder and Reference NIC modules is denoted as FPGA(actual). FPGA(sim) throughput is a theoretical value. It is the upper limit of throughput via $10 \mathrm{GbE}$. The throughput of ChangeFinder module itself, which is derived by the number of cycles, pipeline structure (i.e., interval), and operating frequency of the ChangeFinder module, is $15.6 \mathrm{M}$ packets per second.

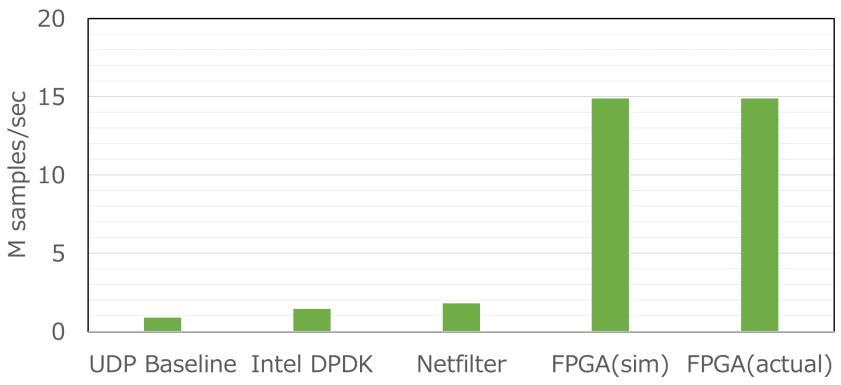

Fig. 14 Throughput of change-point detection [mega samples / sec]

\begin{tabular}{|c|c|c|c|c|}
\hline Ethernet Header & IP Header & UDP Header & Stream ID & Input Data \\
\hline 14Byte & 20Byte & 8Byte & 4Byte & 4Byte
\end{tabular}

Fig. 15 Packet format for the evaluation of multi-stream

FPGA(actual) is the measured throughput using real machines (Table 2). The throughput of FPGA(actual) achieves $16.8 \mathrm{x}$ throughput improvement compared to Baseline. It is much higher than those with software-based optimizations by DPDK and Netfilter. FPGA(actual) and FPGA(sim) are almost the same.

In practical use cases, a specific field of received packets is extracted and fed to ChangeFinder module. In this experiment, we used 46-byte UDP/IP packets containing a single 32-bit float value. This assumption is pessimistic in terms of throughput. sdarl and sdar2 modules accept new data in every eight cycles. Since internal data width of Reference NIC is 256 bits, these sdar modules are not bottleneck when packet length is greater than or equal to 256 bytes. $S_{p}$ denotes the packet length [bits] and $S_{\text {overhead }}$ denotes the total size [bits] of preamble, FCS, and IFG. $T_{\text {actual }}$ denotes the throughput [samples/sec] of FPGA(actual). Ratio against the $10 \mathrm{GbE}$ line rate is denoted as $\mathrm{L}$, and it is calculated as follows.

$$
L=T_{\text {actual }}\left(S_{p}+S_{\text {overhead }}\right) / 10 G[\text { bits } / \mathrm{sec}]
$$

The proposed FPGA(actual) achieves $10 \mathrm{GbE}$ line rate.

We also evaluate the throughput of the multi-stream implementation by RTL simulation. The packet format processed in the NIC is shown in Fig. 15. The format includes a float value and a stream ID which is generated randomly. The throughput was calculated based on the number of clock cycles required to process 10,000 packets. Figure 16 shows their throughputs. Throughput of the multi-stream implementation is sightly lower than the single-stream implementation due to a reduced operating frequency.

\section{Conclusions}

Toward anomaly detection, change-point detection is used to look for change in a probability distribution of time series, while outlier detection is used to look for entity being away from the mean of a probability distribution. ChangeFinder algorithm based on SDAR model supports both the outlier and change-point detections and can be used 


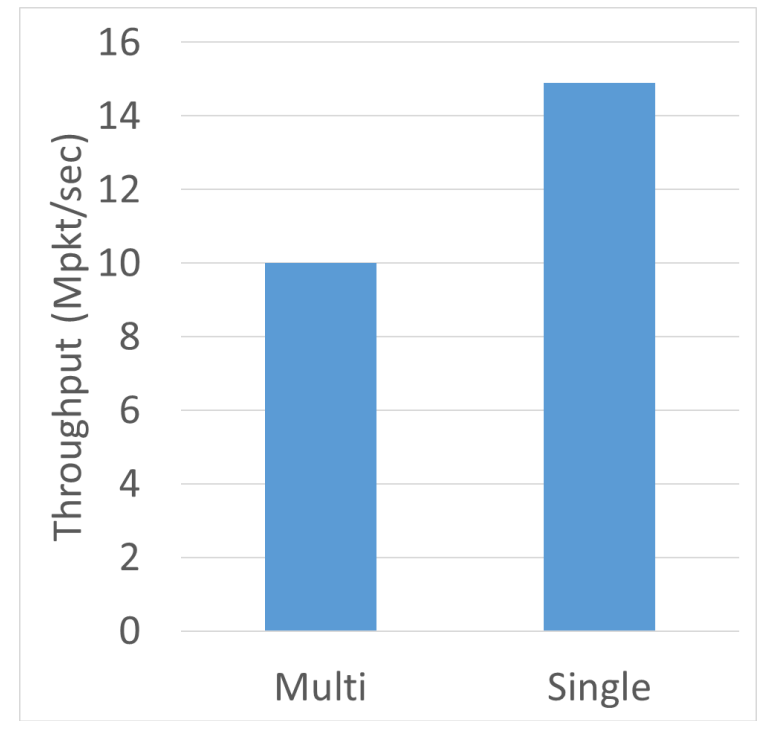

Fig. 16 Throughput evaluation of multi-stream

for online use. This paper is the first work that accelerates ChangeFinder algorithm using FPGA and integrates it into NetFPGA-SUME for high-speed change-point detection at $10 \mathrm{GbE}$ NICs. A single ChangeFinder module can find change-points on many streams coming from the same $10 \mathrm{GbE}$ interface by using a BRAM-based fast context memory. The proposed ChangeFinder NIC is compared to a UDP baseline and two software-based optimizations, i.e., DPDK and Netfilter. The throughput is much higher than these counterparts and it is $16.8 \mathrm{x}$ higher than the UDP baseline. The throughput is corresponding to the $10 \mathrm{GbE}$ line rate. A demonstration video of current design can be found in [25].

\section{Acknowledgments}

This work was supported by JST CREST Grant Number JPMJCR1785, Japan.

\section{References}

[1] H. Wang, D. Zhang, and K.G. Shin, "Change-Point Monitoring for the Detection of DoS Attacks," IEEE Trans. Dependable and Secure Computing, vol.1, no.4, pp.193-208, Oct. 2004.

[2] S. Aminikhanghahi and D.J. Cook, "A Survey of Methods for Time Series Change Point Detection," Knowledge and Information Systems, vol.51, no.2, pp.339-367, May 2017.

[3] V. Guralnik and J. Srivastava, "Event Detection from Time Series Data," Proc. International Conference on Knowledge Discovery and Data Mining (KDD’99), pp.33-42, Aug. 1999.

[4] J. Takeuchi and K. Yamanishi, "A Unifying Framework for Detecting Outliers and Change Points from Time Series," IEEE Trans. Knowl. Data Eng., vol.18, no.4, pp.482-492, 2006.

[5] T. Iwata, K. Nakamura, Y. Tokusashi, and H. Matsutani, "Accelerating Online Change-Point Detection Algorithm using $10 \mathrm{GbE}$ FPGA NIC," Proc. International European Conference on Parallel and Distributed Computing (Euro-Par'18) Workshops, vol.11339, pp.506-517, Aug. 2018.

[6] Y. Urabe, K. Yamanishi, R. Tomioka, and H. Iwai, "Real-Time Change-Point Detection Using Sequentially Discounting Normalized Maximum Likelihood Coding," Proc. Pacific-Asia Conference on Knowledge Discovery and Data Mining (PAKDD’11), vol.6635, pp.185-197, May 2011.

[7] Y. Kawahara and M. Sugiyama, "Change-Point Detection in Time-Series Data by Direct Density-Ratio Estimation," Proc. SIAM International Conference on Data Mining (SDM'09), pp.389-400, April 2009.

[8] K. Yamanishi and J. Takeuchi, "A Unifying Framework for Detecting Outliers and Change Points from Non-Stationary Time Series Data," Proc. International Conference on Knowledge Discovery and Data Mining (KDD’02), pp.676-681, July 2002.

[9] F. Saaid, D. Nur, and R. King, "Change Points Detection of Vector Autoregressive Model using SDVAR Algorithm," Proc. 5th Annual ASEARC Conference, pp.18-21, Feb. 2012.

[10] “Apache Hivemall.” http://hivemall.incubator.apache.org/.

[11] P. Benáček, R.B. Blažek, T. Čejka, and H. Kubátová, "ChangePoint Detection Method on $100 \mathrm{~Gb} / \mathrm{s}$ Ethernet Interface," Proc. ACM/IEEE Symposium on Architectures for Networking and Communications Systems (ANCS'14), pp.245-246, June 2014.

[12] A. Hayashi, Y. Tokusashi, and H. Matsutani, "A Line Rate Outlier Filtering FPGA NIC using 10GbE Interface," ACM SIGARCH Computer Architecture News, vol.43, no.4, pp.22-27, Sept. 2015.

[13] A. Hayashi and H. Matsutani, "An FPGA-Based In-NIC Cache Approach for Lazy Learning Outlier Filtering," Proc. International Conference on Parallel, Distributed, and Network-Based Processing (PDP'17), pp.15-22, March 2017.

[14] Y. Pu, J. Peng, L. Huang, and J. Chen, "An Efficient KNN Algorithm Implemented on FPGA Based Heterogeneous Computing System Using OpenCL," Proc. International Symposium on FieldProgrammable Custom Computing Machines (FCCM'15), pp.167170, May 2015.

[15] P.P. Boopal, M. Garrido, and O. Gustafsson, "A Reconfigurable FFT Architecture for Variable-Length and Multi-Streaming OFDM Standards," Proc. International Symposium on Circuits and Systems (ISCAS'13), pp.2066-2070, May 2013.

[16] T. Ueno, K. Sano, and S. Yamamoto, "Bandwidth Compression of Floating-Point Numerical Data Streams for FPGA-Based HighPerformance Computing," ACM Trans. Reconfigurable Technol. Syst., vol.10, no.3, pp.18:1-18:22, May 2017.

[17] Q. Yun, Y.-H.E. Yang, and V.K. Prasanna, "Multi-Stream Regular Expression Matching on FPGA," Proc. International Conference on Reconfigurable Computing and FPGAs (ReConFig'11), pp.86-91, Nov. 2011.

[18] Y. Qu, Y.-H.E. Yang, and V.K. Prasanna, "Large-Scale Multi-Flow Regular Expression Matching on FPGA," Proc. International Conference on High Performance Switching and Routing (HPSR'12), pp.70-75, June 2012.

[19] N. Zilberman, Y. Audzevich, G.A. Covington, and A.W. Moore, "NetFPGA SUME: Toward 100 Gbps as Research Commodity," IEEE Micro, vol.34, no.5, pp.32-41, Sept. 2014.

[20] “The NetFPGA Project." http://netfpga.org/.

[21] Xilinx, AXI Reference Guide, 2011.

[22] I. Sharafaldin, A.H. Lashkari, and A.A. Ghorbani, "Toward Generating a New Intrusion Detection Dataset and Intrusion Traffic Characterization," Proc. 4th International Conference on Information Systems Security and Privacy, pp.108-116, 2018.

[23] A.G. Tartakovsky, B.L. Rozovskii, R.B. Blazek, and H. Kim, "A novel approach to detection of intrusions in computer networks via adaptive sequential and batch-sequential change-point detection methods," IEEE Trans. Signal Process., vol.54, no.9, pp.3372-3382, 2006.

[24] “TOPIX historial price." https://quotes.wsj.com/index/JP/XTKS/ I0000/historical-prices.

[25] YouTube, "Accelerating ChangeFinder using 10Gbps FPGA NIC." https://www.youtube.com/watch?v=wgTcBfkE5hY. 

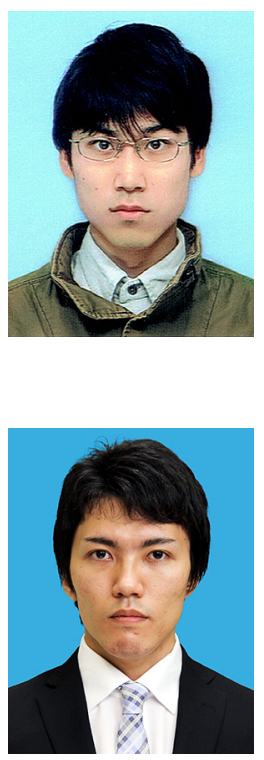

Kohei Nakamura received the BE and ME degrees from Keio University in 2016 and 2018, respectively.
Takuma Iwata received the BE degree from Keio University in 2018 . He is currently a master course student in Keio University.
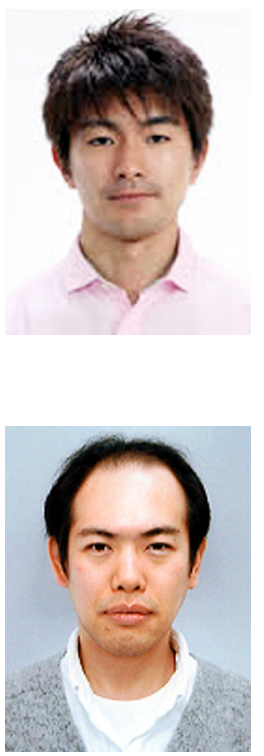

Yuta Tokusashi received the BA, ME and $\mathrm{PhD}$ degrees from Keio University in 2014, 2016, 2019, respectively. He is currently a research associate at the Computer Laboratory in the University of Cambridge. His current research interests include computer architecture and FPGA systems.

Hiroki Matsutani received the BA, ME, and $\mathrm{PhD}$ degrees from Keio University in 2004, 2006, and 2008, respectively. He is currently an associate professor in the Department of Information and Computer Science, Keio University. His research interests include the areas of computer architecture and interconnection networks. 